Galindo, Miguel-Ángel y Méndez, María Teresa. La actividad emprendedora y competitividad: factores que inciden sobre los emprendedores.

\title{
LA ACTIVIDAD EMPRENDEDORA Y COMPETITIVIDAD: FACTORES QUE INCIDEN SOBRE LOS EMPRENDEDORES
}

\section{ENTREPRENEURSHIP ACTIVITY AND COMPETITIVENESS: ENTREPRENEURSHIP ENHANCE FACTORS}

\author{
MIGUEL-ÁNGEL GALINDO MARTÍN \\ Catedrático de Universidad \\ Universidad de Castilla-La Mancha \\ mgalindomar@orange.es
}

\author{
MARÍA TERESA MÉNDEZ PICAZO \\ Profesora Contratada Doctor \\ Universidad Complutense de Madrid \\ mmendezpi@ccee.ucm.es
}

Fecha recepción: 8 de octubre de 2010 Fecha aceptación: 29 de abril de 2011 doi: 10.5209/rev_PADE.2011.v22.3

\begin{abstract}
Competitiveness in a broad sense is based on a series of pillars in which entrepreneurships play an important role. Due to this fact together with the positive relationship between entrepreneurship and economic growth it is necessary to design appropriate measures to encourage such activity. In this article, in addition to presenting entrepreneurship characteristics, we analyze the effects that fiscal and monetary policies on entrepreneurships.
\end{abstract}

KEYWORDS: competitiveness, entrepreneurship, economic growth, fiscal policy, monetary policy

JEL: L26, O40, E62, E63.

\section{RESUMEN}

La competividad en un sentido amplio se fundamenta en una serie de pilares en los que los emprendedores desempeñan un papel importante. Ello junto con la relación positiva que guardan con el crecimiento económico hace que sea necesario que se diseñen medidas adecuadas para estimular dicha actividad. En este artículo, además de exponer las características del emprendedurismo, analizaremos los efectos que ejercen las políticas fiscal y monetaria sobre los emprendedores.

PALABRAS CLAVE: competitividad, emprendedores, crecimiento económico, política fiscal, política monetaria 
Galindo, Miguel-Ángel y Méndez, María Teresa. La actividad emprendedora y competitividad: factores que inciden sobre los emprendedores.

\section{INTRODUCCIÓN}

Desde hace algunas décadas, la eficacia de una determinada política se ha medido mediante el crecimiento económico que generaba. Así, se supone que las naciones que presentan mayores niveles de crecimiento están haciéndolo mejor. Además, sirven de ejemplo a aquéllas que no presentan la misma tendencia y niveles.

Por ello se ha desarrollado una extensa literatura para tratar de delimitar los factores que favorecen ese crecimiento económico, ya que a través de él se mantiene o aumenta el empleo y se produce una mayor cantidad de bienes y servicios, lo que redunda en un bienestar más elevado para la sociedad. Con el paso del tiempo y gracias al avance producido en las técnicas econométricas y en los datos por países, se han podido ir introduciendo distintos tipos de variables, tanto cuantitativas como cualitativas, para tener una visión más completa de la cuestión.

Sin haber alcanzado conclusiones definitivas, nos encontramos con una importante crisis económica en la cual, junto a la discusión ideológica sobre qué tipo de política habría que implantar, se ha concedido la máxima importancia al objetivo de crecimiento económico.

Junto a este objetivo esencial, se han añadido otros que serían la base sobre la que tendría que apoyarse dicho crecimiento, como es la necesidad de reformas estructurales y la competitividad. En el caso de este último, como veremos, se le considera desde una perspectiva más amplia que la tradicional, contemplando una serie de factores que hay que tener en cuenta para poder mejorar dicha competitividad, cuya finalidad es aumentar el nivel de renta y bienestar de los ciudadanos, lo mismo que pretende el crecimiento económico.

Pero para poder llevar a cabo esta tarea hace falta no sólo la actuación de los decisores políticos, proporcionando entre otras cuestiones el marco legal y legislativo adecuado para llevar a cabo las actividades, sino también personas que estén dispuestas a crear o ampliar sus negocios. Dentro de este segundo grupo tenemos a los emprendedores, que constituyen el núcleo esencial de nuestro análisis.

Así pues, el objetivo del artículo es analizar los factores que afectan a la actividad emprendedora como factor de crecimiento y competitividad. Se expondrán las medidas que pueden incentivarla, así como la relación que guarda la misma con algunos de los pilares de la competitividad. Para ello, en el epígrafe 2 delimitaremos la competitividad en un sentido amplio, en el tercero nos referiremos a las características de los emprendedores, en el cuarto nos referiremos a la relación entre emprendedores y crecimiento económico, los factores que inciden sobre éstos, en el quinto expondremos las medidas que estimulan la actividad emprendedores y en el último recogeremos las principales conclusiones.

\section{COMPETITIVIDAD EN SENTIDO AMPLIO}

Cuando se habla de competitividad, habitualmente se suele hacer referencia a la capacidad que tienen los países para aumentar de una forma sostenida la presencia de sus productos en los mercados internacionales e internos, de tal forma que van a 
Galindo, Miguel-Ángel y Méndez, María Teresa. La actividad emprendedora y competitividad: factores que inciden sobre los emprendedores.

ser los precios y los costes relativos los que desempeñan un papel esencial en este tipo de análisis. Tal es la idea que subyace en la definición que proporciona por ejemplo la OCDE (1992) sobre competitividad, aunque añadiendo que a su vez se consigue mantener y aumentar a largo plazo los ingresos reales de los habitantes del país en cuestión.

Sin embargo existe una perspectiva más amplia sobre dicho concepto en la que, como señala Matilde Madrid (2010), se amplía el ámbito puramente comercial considerando también una perspectiva a largo plazo, que se suele asociar al bienestar, lo que supone contemplar el concepto de competitividad agregada que está vinculado con la productividad y con los factores que afectan a la eficiencia productiva. Desde esta perspectiva, nos encontramos con que la competitividad estaría vinculada con aspectos tales como la capacidad de innovar, mercados eficientes, dotación y utilización de factores productivos y las características de la organización empresarial, pero por otro lado, estos actores estarían a su vez relacionados con otros, tales como la calidad de la educación, la dotación de infraestructuras o la capacidad de organización y gestión de las empresas, esto es, de factores que generan un entorno o clima favorable para las empresas (Madrid, 2010, p. 93).

Este tipo de competitividad es la que analiza el World Economic Forum (WEF) desde finales de la década de los 70 del pasado siglo, que la define como el conjunto de instituciones, políticas y factores que determinan el nivel de productividad de un país (World Economic Forum, 2010, p. 4). Ello significa, desde el punto de vista de este informe, que los países más competitivos son los que son capaces de generar unos mayores niveles de renta para sus ciudadanos.

En concreto el WEF señala doce pilares que sostienen la competitividad de los países y que son los siguientes (World Economic Forum, 2010, pp. 4-8):

1) Las instituciones, esto es, el marco legal y administrativo en el que los agentes económicos llevan a cabo su actividad para generar renta y bienestar.

2) Infraestructuras, que facilitan el buen funcionamiento de la economía reduciendo las distancias entre las regiones y determinando la localización de la actividad económica.

3) Entorno macroeconómico, ya que la estabilidad macroeconómica es un estímulo para llevar a cabo actividades empresariales.

4) Salud y educación primaria, ya que la salud es necesaria para que los trabajadores puedan realizar sus tareas y la educación para mejorar su eficiencia.

5) Educación superior y formación, imprescindibles para implantar procesos de producción más sofisticados. Ambas facilitan el catch-up tecnológico, que es un factor importante en el proceso de convergencia económica.

6) Mercados de bienes eficientes, ya que facilitan la elaboración de los bienes y servicios que se necesitan y aseguran su comercialización eficiente en la economía.

7) Mercado de trabajo eficiente, que asegura la adecuada asignación de la mano de obra y proporciona los incentivos adecuados a los trabajadores para que lleven a cabo sus tareas de una forma más efectiva. 
Galindo, Miguel-Ángel y Méndez, María Teresa. La actividad emprendedora y competitividad: factores que inciden sobre los emprendedores.

8) Desarrollo del mercado financiero, para asignar de forma eficiente los ahorros generados por los individuos y facilitar los recursos a aquéllos que deseen crear nuevos negocios o expansionar o mejorar los existentes.

9) Implantación de tecnología, lo que permite a la economía adoptar aquellas tecnologías que mejoran la actividad de la mano de obra.

10) Tamaño del mercado, ya que los de mayor tamaño permiten a las empresas aprovechar las economías de escala.

11) Sofisticación de los negocios, es decir, la calidad de los negocios que se llevan a cabo en el país, así como la de las operaciones y estrategias que realizan los individuos. Cuanto mayor sea dicha sofisticación, más elevada será la competitividad.

12) Innovación, al igual que lo que se señalaba en el punto 9, porque a través de ella se mejora la eficiencia de los trabajadores.

Todos estos pilares están interrelacionados, de tal manera que algunos de ellos refuerzan la actividad de los otros. Por ejemplo, sin una educación y formación adecuadas resulta muy complicada la implantación de las innovaciones tecnológicas, que a su vez favorecen una mayor sofisticación de los negocios que se están llevando a cabo.

Teniendo en cuenta lo señalado hasta este momento, uno de los factores que juega un papel importante en el ámbito de la competitividad sería el emprendedor, ya que no sólo generan nuevos negocios que conllevarían una mayor renta y bienestar para los ciudadanos, sino que también llevan a cabo procesos de implantación de tecnologías y generan innovaciones. Es por ello que tenemos que delimitar qué se entiende por emprendedor.

\section{EMPRENDEDORES: CONCEPTO Y CARACTERÍSTICAS}

A la hora de referirnos al término "entrepreneurship" la principal dificultad con la que nos encontramos es su definición, ya que no existe una de carácter general y globalmente aceptada. Así, por ejemplo, Wennekers y Thurik (1999) siguiendo las ideas expuestas por Hebert y Link (1989), Bull y Willard (1993) y Lumpkin y Dess (1996), lo conceptualizan como la capacidad manifiesta y deseo de los individuos, ya sea por ellos mismos o mediante equipos, dentro o fuera de organizaciones existentes de crear nuevas oportunidades económicas, esto es, nuevos productos, nuevas formas de organización, nuevos métodos de producción, etc., e introducir sus ideas en los mercados, haciendo frente a la incertidumbre y a otros obstáculos, adoptando decisiones sobre la localización y en la forma y uso de los recursos y de las instituciones.

Por tanto, en términos generales, las características de dicha definición serían las siguientes:

1) Se hace hincapié en el aspecto del comportamiento característico de los agentes económicos. Por tanto, a la hora de hablar de emprendedores no nos estamos refiriendo a una ocupación, sino a una actividad, en la que se tienen en cuenta los aspectos y circunstancias que rodean al individuo.

2) Los emprendedores deben tener en cuenta la incertidumbre y otros obstáculos inherentes a la actividad que desempeñan. 
Galindo, Miguel-Ángel y Méndez, María Teresa. La actividad emprendedora y competitividad: factores que inciden sobre los emprendedores.

3) Deben disponer de información sobre los diferentes procesos de producción que sean eficientes, así como de las nuevas formas de organización. Dicha información puede adquirirse en centros de formación o a través de expertos.

4) La actividad emprendedora no debe asociarse sólo a las pequeñas empresas. Este tipo de emprendedores también se encuentra en las grandes empresas, y se les suele denominar "intrapreneurs" o "corporate entrepreneurs".

Desde una perspectiva moderna, podemos considerar tres tipos de emprendedores:

1) Innovador, de acuerdo con el planteamiento defendido por Schumpeter $(1911,1950)$. Este autor considera que la actividad emprendedora supone innovación en lo que se refiere a la introducción de un nuevo producto, en la organización o proceso, y supone un proceso de destrucción. El emprendedor crea nuevas industrias y por ello provoca cambios estructurales importantes en la economía. Pero no puede ser considerado puramente como inventor, sino que lo que hace es introducir las invenciones creadas por otros. Es por ello que Schumpeter considere al emprendedor como un innovador que destruye las estructuras existentes. Desde esta perspectiva, se hace hincapié en el pilar 9 señalado anteriormente, así como también en el 11. Asimismo, hay que tener en cuenta que lo señalado no implica necesariamente que se tenga que crear siempre un nuevo producto, sino que se puede desarrollar una actividad que desarrolle productos ya existentes en el mercado, empleando para ello nuevos avances tecnológicos.

2) Aprovechar las oportunidades de beneficio (Kirzner, 1973, 1999). Kirzner, al igual que Schumpeter, considera que el emprendedor trata de aprovecharse de las oportunidades de beneficio que proporcionan los mercados, pero a diferencia de aquél, considera también que aprende de los errores pasados e intenta corregirlos, conduciendo de esta manera al mercado hacia un nuevo equilibrio. Por lo tanto, desde su perspectiva, existe una relación entre instituciones y emprendedores que incentiva el progreso económico a través de dos factores. En primer lugar, las instituciones proporcionan el nivel de competitividad que los emprendedores necesitan, y en segundo lugar, también proporcionan la estructura de incentivos que es necesaria para estimular la actividad emprendedora. En definitiva, esta aportación hace hincapié en el primer pilar de los señalados en el apartado anterior.

3) Incertidumbre (Knight, 1921). Knight considera que la incertidumbre es un factor importante que tienen que tener en cuenta los emprendedores, ya que tienen que tomar sus decisiones en un mundo incierto, y sus beneficios se van a ver afectados por dicha incertidumbre. En este caso, se presta especial atención al tercer pilar, referente al entorno macroeconómico, ya que cuanto más estable sea menos incertidumbre se genera.

4) La diferenciación de la actividad emprendedora realizada por Baumol (1990), que distingue entre "productivo" y "no productivo". Desde su punto de vista, gran parte de los emprendedores son creativos e ingeniosos, y buscan los medios más adecuados para aumentar su riqueza, poder y prestigio, viéndose afectados por el entorno que les rodea. En este caso, se hace hincapié no sólo en el tercer pilar, sino también en el decimoprimero, referido a la sofisticación de los negocios. 
Galindo, Miguel-Ángel y Méndez, María Teresa. La actividad emprendedora y competitividad: factores que inciden sobre los emprendedores.

Finalmente, esta enumeración se completaría desde el punto de vista sociológico, con las ideas expuestas por Weber $(1978,1988)$. De acuerdo con Swedberg (2000) se podrán considerar tres características inherentes al emprendedor:

a) Carisma. Weber consideraba que el emprendedor es un tipo de persona que averigua lo que necesitan otras personas y así consigue que le sigan. De esta forma, coloca sus productos en el mercado y consigue los trabajadores necesarios para desarrollar la actividad.

b) Religión. En su conocido The Protestant Ethic and the Spirit of Capitalism, Weber consideraba que un determinado tipo de religión que denominaba "ascetic Protestantism" favorecía la generación de aptitudes positivas para la obtención de dinero, que a su vez propiciaba la aparición de empresarios.

Así pues, a partir de la exposición realizada en este apartado comprobamos que existe una importante relación entre los emprendedores y algunos de los pilares en los que se apoya la competitividad.

\section{EMPRENDEDORES, CRECIMIENTO ECONÓMICO Y COMPETITIVIDAD}

Una vez expuestas las características del emprendedor, vamos a exponer su relación con el crecimiento económico. La importancia de ello radica en el papel que desempeña en la actualidad el crecimiento económico como objetivo de política económica.

En este sentido, cabe señalar que desde las últimas décadas del siglo pasado, se ha venido evaluando la bondad 0 no de una determinada política a través del crecimiento que se venía alcanzando. Aquellas naciones que presentan mayores niveles de crecimiento son consideradas como las que mejor política económica están desarrollando y son dignas de elogio convirtiéndose en un modelo a seguir por aquellas otras que muestran unos niveles más bajos.

Ello se debe, entre otras cosas, al hecho de que un mayor crecimiento supone un bienestar más elevado para la sociedad, ya que no sólo supone disponer de un mayor número de bienes y servicios a disposición de los ciudadanos, sino también mantener e incluso aumentar los puestos de trabajo, imposibilitando, por tanto, que aumente el desempleo, lo que supone a su vez paz social. Así pues, entramos en un círculo "virtuoso" en el que tenemos que encontrar aquellos factores que potencian el crecimiento, ya que con ello a su vez mejoramos el bienestar y el clima sociales, lo que a su vez favorecerá crecimientos futuros.

A la hora de potenciar el crecimiento económico, ya hemos indicado que se han venido considerando distintas variables, tanto económicas como sociales. Una de ellas es el papel que tienen los emprendedores en este proceso. En principio, dicha relación parece obvia, ya que un país en el que no existan personas que estén dispuestas a elaborar bienes y servicios arriesgando sus recursos difícilmente podría crecer de una forma sostenida. Las situaciones caracterizadas por la existencia de ineficiencia económica son un incentivo para la aparición de personas que estén dispuestas a aprovechar mejor los recursos, conduciendo a la economía hacia otra situación más eficiente, aumentando de esta manera las posibilidades de producción y utilizando en mayor medida los recursos disponibles. Todo ello representa la 
Galindo, Miguel-Ángel y Méndez, María Teresa. La actividad emprendedora y competitividad: factores que inciden sobre los emprendedores.

esencia del crecimiento, ya que a través de un incremento de la productividad, aumenta el producto real de la economía.

Pero también hay que tener en cuenta el efecto indirecto expuesto por Holcombe (1998), según el cual, el comportamiento de un determinado emprendedor, si tiene éxito, no sólo motiva a otros a seguir su ejemplo, sino que también crea nuevas oportunidades para que las aprovechen terceros.

Obviamente, para que este proceso se desarrolle, debe existir el adecuado clima o entorno en el que se pueda llevar a cabo esta actividad. Como se expondrá más adelante, resulta necesario un entramado legal que proteja la propiedad privada, un determinado grado de libertad, la posibilidad de realizar los acuerdos necesarios que faciliten las operaciones comerciales, la existencia de una "rule of law", etc. (Harper, 1998, North, 1994, Olson, 1996).

Ahora bien, hay que tener presente que si existe una conexión entre emprendedurismo y crecimiento económico, también se produce a la inversa, esto es, nos encontramos ante un efecto "feed-back", que a veces se suele olvidar en los análisis y que también tiene una gran relevancia. Asimismo, en este contexto también hay que tener en cuenta otra serie de factores que influyen en ambos y que condicionan los resultados obtenidos. Todas estas relaciones se recogen en la figura-1 que exponemos a continuación.

En ella podemos comprobar cómo las circunstancias socioeconómicas, la cultura y la evolución del país, afectan a la capacidad emprendedora.

Figura-1

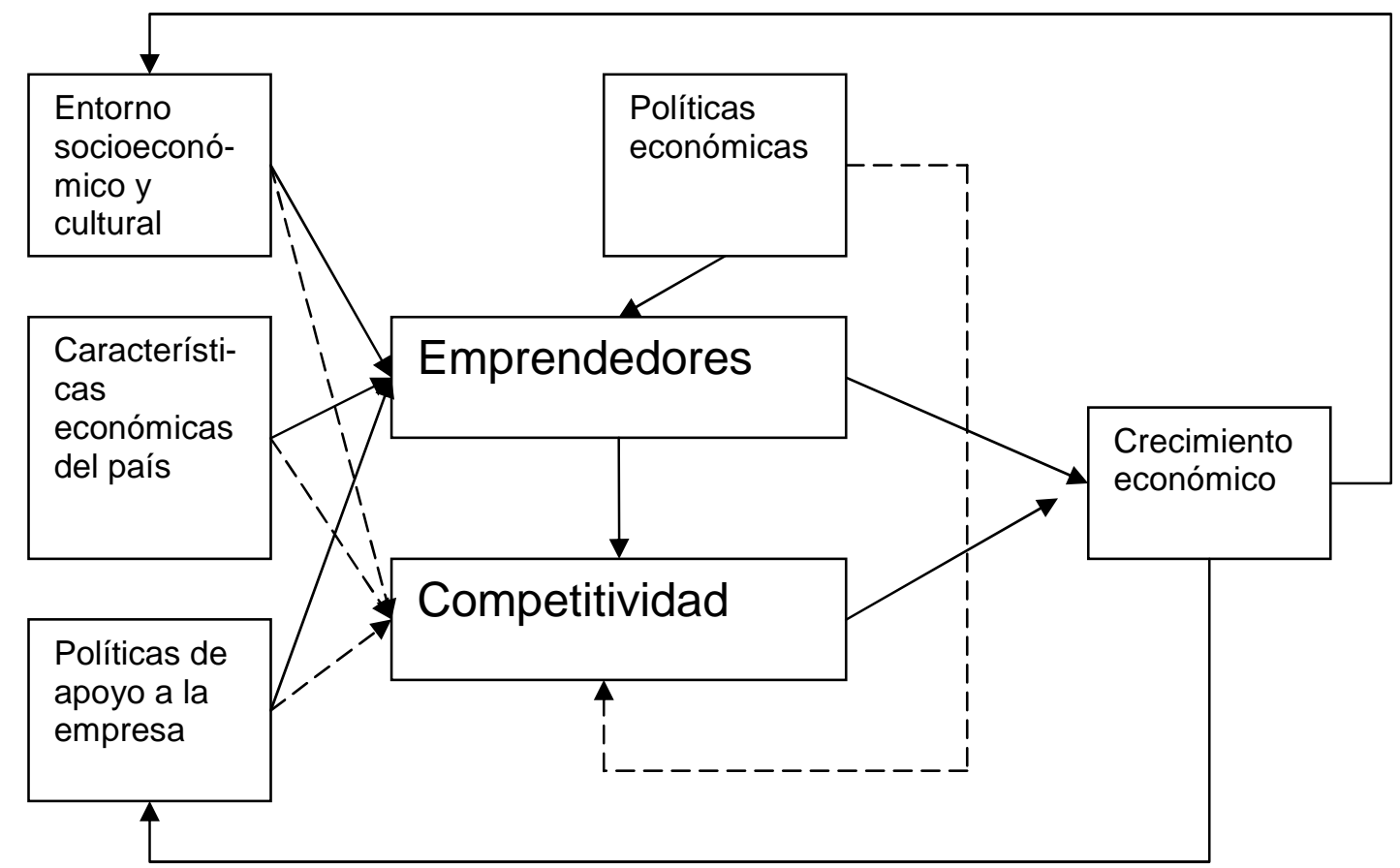

Fuente: Elaboración propia 
Galindo, Miguel-Ángel y Méndez, María Teresa. La actividad emprendedora y competitividad: factores que inciden sobre los emprendedores.

En este sentido, son diversos los aspectos que tenemos que considerar. En primer lugar, debe existir un clima favorable hacia el emprendedor.

En segundo lugar, el entorno familiar y social del posible emprendedor también es objeto de consideración. Su formación, el clima en el que ha desarrollado su vida, etc., hacen que exista una mayor o menor propensión a realizar una actividad emprendedora. En algunos países, a pesar de que los individuos alcanzan un elevado grado de formación a través de las universidades, se muestran reacios a llevar a cabo actividades empresariales, conformándose con ser asalariados, ya que consideran, entre otras cuestiones, que ello entraña un menor riesgo y que van a tener más o menos garantizado su salario.

En tercer lugar, en relación a los dos puntos anteriores, hay que considerar también la cultura existente en el país. Esta ejercería un efecto positivo sobre el emprendedor, ya que no sólo le permitiría asimilar mejor los avances tecnológicos e introducirlos en su negocio, sino también prepararse mejor ante las circunstancias cambiantes en el entorno económico y afrontar de una manera más efectiva las alteraciones que se pudieran producir. Ese nivel cultural también ejerce un efecto positivo por el lado de los trabajadores, ya que al igual que al empresario, les motiva a aceptar mejor los cambios tecnológicos y a propiciar su introducción, lo que redundará en un mayor crecimiento económico. Pero frente a estos efectos positivos, este clima cultural también presenta desventajas. Estas se pueden concretar, por un lado, en los sistemas de protección de los trabajadores, que supondrían un coste que podría ser inaceptable para los posibles emprendedores y restaría competitividad a las empresas. Por otro lado, como ya hemos indicado anteriormente, puede motivar a los individuos a ser empleados más que emprendedores.

En cuarto lugar, también influyen en este proceso las expectativas sobre la actividad económica del país. En un contexto en el que existen unos buenos resultados económicos y perspectivas de crecimiento, el número de emprendedores debería aumentar ante la posibilidad de encontrar nuevos nichos de mercado, lo que redundaría en una mejoría de su cuenta de resultados. Ello significa, por tanto, que la introducción de nueva tecnología, así como el comportamiento demográfico y de las instituciones, generan una serie de oportunidades por el lado de la demanda que los emprendedores deben aprovechar. Obviamente, el resultado de ello depende también de las capacidades, habilidades y cultura que posean.

A lo indicado hay que añadir que, de acuerdo con lo expuesto en los apartados anteriores, todos estos factores también afectarían a la competitividad del país, incidiendo ambos, emprendedores y competitividad, sobre el crecimiento económico.

Por otro lado, hay que tener presente que el sector exterior también afectaría a los emprendedores, pero desde una perspectiva tanto positiva como negativa. Desde la vertiente negativa, un proceso de apertura exterior en la que se supriman barreras y se permita la libre circulación de personas y bienes, supone poder aumentar el volumen de negocio, ya que se puede acudir a esos nuevos mercados y a su vez contratar a aquellos trabajadores que estén más cualificados. Pero a su vez ello tiene como efecto compensador el hecho de que los productos deben ser competitivos, lo que significa que la política económica diseñada debe ser no 
Galindo, Miguel-Ángel y Méndez, María Teresa. La actividad emprendedora y competitividad: factores que inciden sobre los emprendedores.

inflacionista y a su vez los costes salariales deben ser moderados. En este orden de cosas, hay que tener presente también la competencia que supone dicha apertura, ya que pueden introducirse en nuestra economía empresas extranjeras con nueva tecnología, lo que perjudicaría la aparición de nuevos emprendedores. En este sentido, la inversión directa extranjera tendría efectos tanto positivos como otros que habría que tratar de reducir.

Finalmente, hay que añadir también que cierta actividad emprendedora podría suponer intentos de concentración empresarial, favorecidos por el proceso innovador que algunos empresarios pudieran desarrollar. Como acabamos de indicar, ante la aparición de inversiones extranjeras muy competitivas, las pequeñas y medianas empresas de los países pueden resentirse en su volumen de negocio y desaparecer. Ello provocaría la implantación de políticas económicas que favoreciesen a esas empresas. En este sentido, también se podría considerar la necesidad de aplicar políticas fiscales que favoreciesen a los emprendedores a la hora de desarrollar su actividad. Ello tendría dos efectos contrarios. Por un lado un efecto positivo, ya que si son efectivas, generarían una mayor actividad productiva. Aquí, cabría encuadrar las políticas tendentes a mejorar las infraestructuras del país, las políticas de I+D+i, etc. Pero por otro lado, hay que tener presentes también los efectos negativos derivados de su financiación, que supondría un detrimento de los recursos destinados a la producción, así como otros, como el tipo de interés, un mayor endeudamiento, efectos "crowding-out", riqueza, etc. En definitiva, el resultado final con este tipo de medidas no queda totalmente claro.

Por tanto, hemos podido comprobar la existencia de un número importante de medidas a través de las cuales se podría potenciar la actividad de los emprendedores, lo que redundaría en un mayor crecimiento económico. Pero como ya anticipamos, el proceso no finaliza aquí, ya que este crecimiento afectaría a las condiciones que inciden sobre el emprendedor, lo que podría incentivar la aparición de nuevos empresarios.

En efecto, el crecimiento económico supone cambios culturales y sociales que hacen que la visión del emprendedor cambie, así como sus expectativas respecto al futuro. Una sociedad con mayor bienestar viene acompañada de mayores propensiones a consumir, lo que hace que los nichos de mercado aumenten. Los emprendedores captan los deseos de los agentes económicos deseosos de satisfacer las cada vez mayores necesidades que genera la sociedad de consumo. El proceso innovador, a su vez, les facilita la posibilidad de crear nuevos productos que hagan obsoletos los anteriores. A pesar de que, como hemos indicado anteriormente, el grado de competencia aumenta debido a la apertura de mercados, aquellos que analizan y conocen mejor los mercados son los que tienen mayores posibilidades de sobrevivir y aumentar sus beneficios.

El conocimiento de los individuos también resulta importante para desarrollar su actividad. Se pretende ser original y a su vez mostrar interés por el bienestar de la sociedad. El marketing y la publicidad ayudan a su vez, pues introducen nuevos elementos que se consideran socialmente deseables, como por ejemplo, el carácter ético del negocio. Todo ello es posible gracias al bienestar que ha generado el crecimiento económico y a los efectos demostración que se derivan del mismo. Así 
Galindo, Miguel-Ángel y Méndez, María Teresa. La actividad emprendedora y competitividad: factores que inciden sobre los emprendedores.

pues, podemos señalar un efecto "feed-back" en el proceso, que hay que conocer y tener presente en el análisis.

\section{MEDIDAS QUE AFECTAN A LA ACTIVIDAD EMPRENDEDORA}

En el apartado anterior hemos indicado el efecto positivo que tiene el emprendedurismo sobre el crecimiento económico. Ello viene corroborado por los diversos análisis empíricos que señalan la relación positiva entre ambas variables (véanse por ejemplo Acs, Audretsch, Braunerhjelm y Carlson, 2004, 2005; Stel, Carree y Thurik, 2005; Audretsch, 2006 y 2009; Audretsch, Bonte y Keilbach, 2008; Audretsch y Keilbach, 2004a,b, 2008; y Galindo, Méndez y Alfaro, 2010, entre otros).

Por lo que se refiere a la evolución del emprendedurismo en el caso de algunos países europeos, ésta viene reflejada en las figuras 2, 3 y 4 que exponemos a continuación. Los datos corresponden al índice Total Enterpreneurship Activity (TEA) elaborado por el Global Entrepreneurship Monitor (GEM) que muestra el porcentaje de actividad emprendedora sobre la población adulta de un país o región. Se obtiene de agregar el porcentaje de población adulta involucrada en iniciativas nacientes y nuevas.

Como se puede comprobar en las figuras, el inicio de la crisis ha supuesto una reducción del TEA, que tras las medidas que se han adoptado en algunos de los países, parece que ha permitido que en el último año, 2008, la tendencia haya cambiado y se muestre creciente con respecto al año anterior. Pero según como vayan evolucionando las economías y se vayan resolviendo los problemas que está generando la crisis económica, dicha tendencia creciente puede mantenerse o por el contrario, frenarse y cambiar, con los efectos negativos que ello supone para el crecimiento económico y el empleo, entre otras cuestiones.

Es por ello que sea necesario adoptar medidas y actuaciones que incentiven la actividad emprendedora. Y a ellas nos vamos a referir a continuación.

Figura-2: Total Enterpreneurship Activity (TEA) 2002-2008

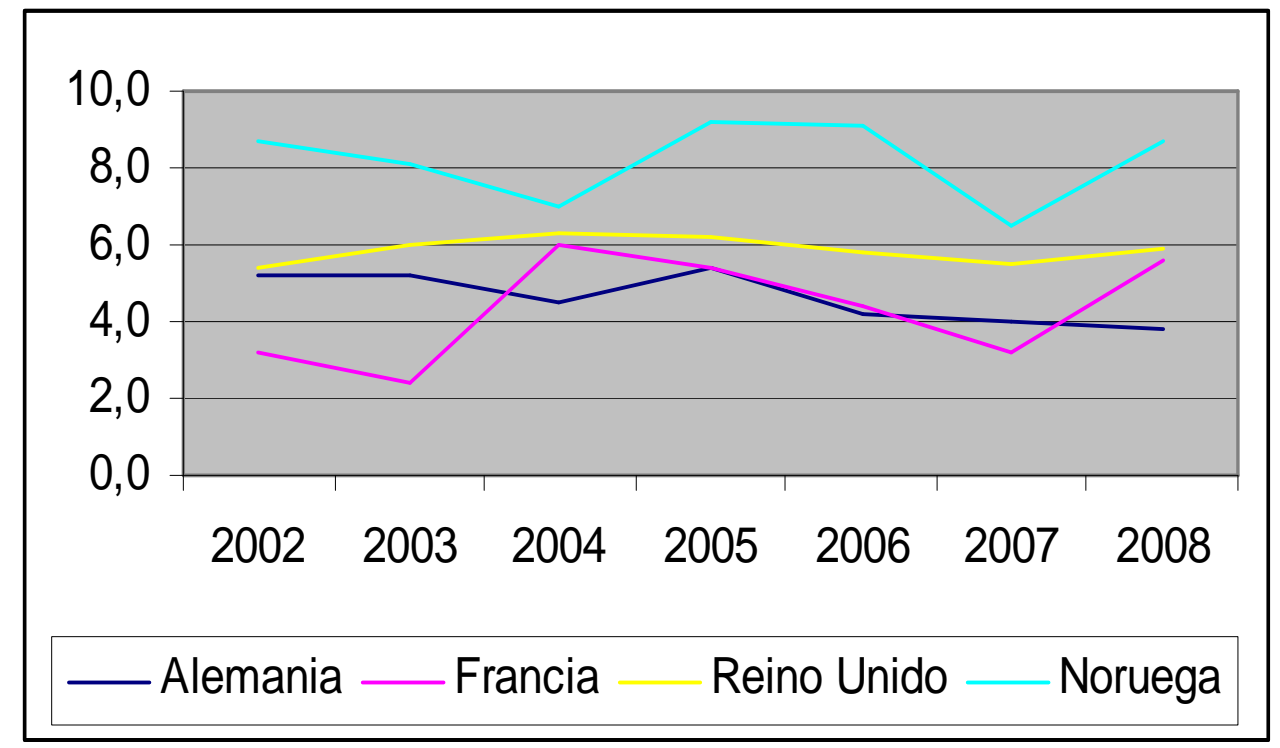

Fuente: Global Entrepreneurship Monitor (GEM) 
Galindo, Miguel-Ángel y Méndez, María Teresa. La actividad emprendedora y competitividad: factores que inciden sobre los emprendedores.

Figura-3: Total Enterpreneurship Activity (TEA) 2002-2008

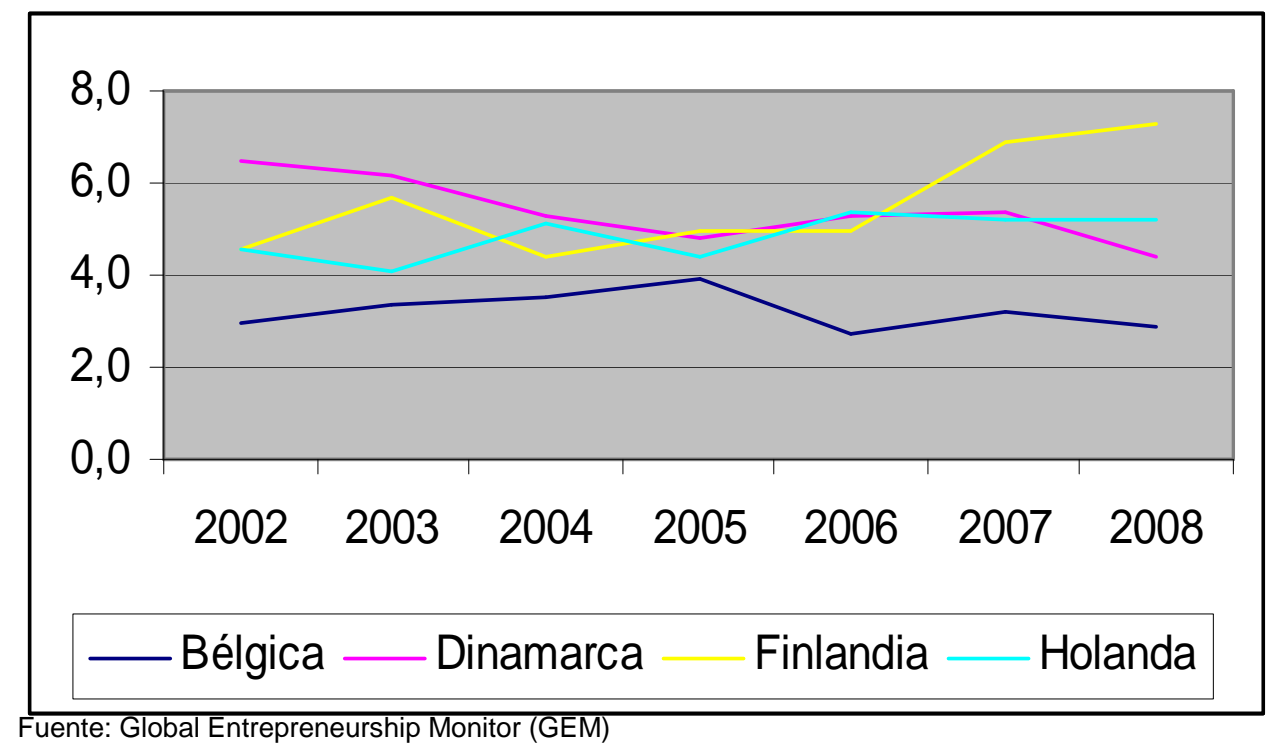

Figura-4: Total Enterpreneurship Activity (TEA) 2002-2008

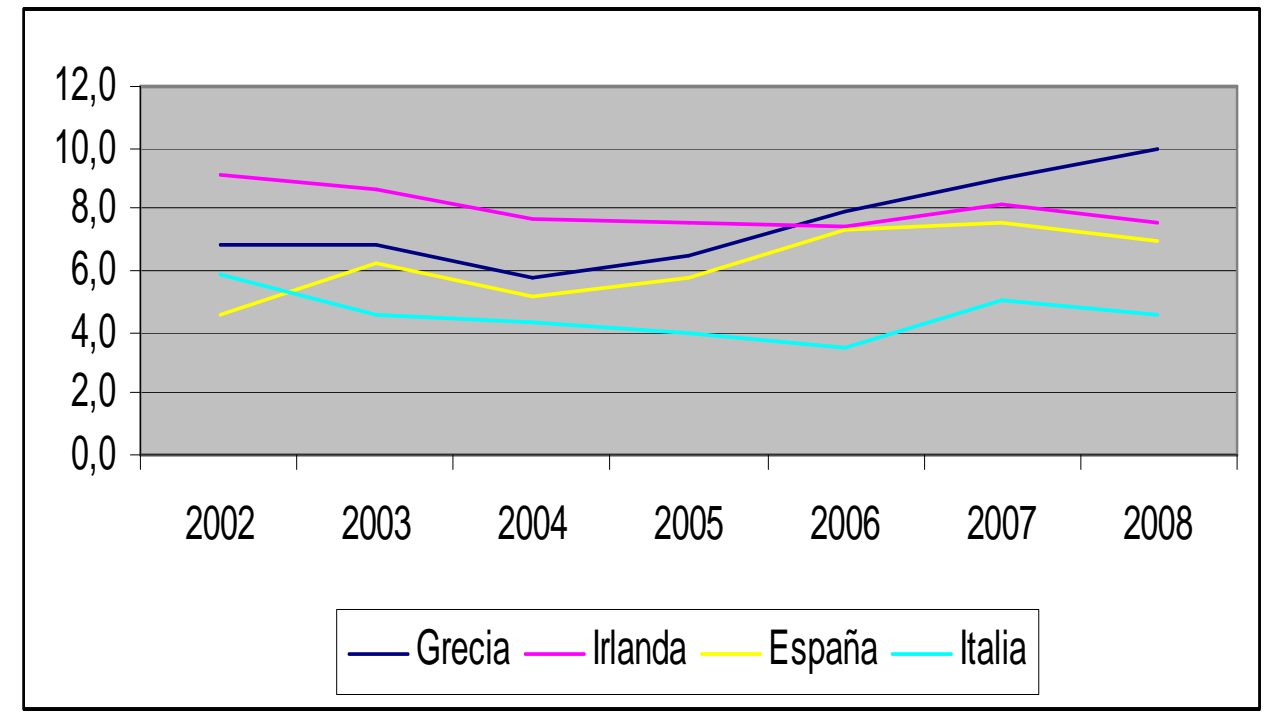

Fuente: Global Entrepreneurship Monitor (GEM)

En este sentido, hay que comenzar señalando que existen trabajos en los que se recogen algunas variables cualitativas a través de las cuales se incidiría sobre la actividad emprendedora, como es el caso del capital social (Lin, 2001, Birley, 1985, M. Bahmani-Oskooee, Galindo y Méndez, 2008 y S. Bahmani, Galindo y Méndez, 2011), y las organizaciones sin fines de lucro (M. Bahmani, Galindo y Méndez, 2011), entre otras.

Pero en este caso nos vamos a centrar básicamente en las políticas fiscal y monetaria. Por lo que se refiere a la primera de ellas, uno de sus efectos positivos se produciría a través de políticas de mejora de infraestructuras, ya que supone no sólo acercar los mercados, sino también ahorrar costes a los emprendedores, tanto en tiempo como en transporte. En la estimación realizada por Alfranca, Galindo y Méndez (2011) para el caso de 11 países de la OCDE, se muestra una relación positiva entre ambas variables. Lo mismo sucede si se considera no el caso del TEA 
Galindo, Miguel-Ángel y Méndez, María Teresa. La actividad emprendedora y competitividad: factores que inciden sobre los emprendedores.

total, sino el que corresponde sólo a las mujeres (M. Bahmani, Galindo y Méndez, 2011).

Una segunda actuación a considerar por parte de la política fiscal sería a través de los impuestos. Una reducción del impuesto que recae sobre la renta supondría, por un lado, un aumento de la renta disponible, lo que conduciría a un mayor consumo que estimularía a los emprendedores a aumentar su actividad y, por otro, menores impuestos a pagar por ellos, los que recaen sobre las empresas, teniendo el mismo efecto positivo. En el análisis empírico efectuado en Galindo, Méndez y Alfaro (2010) se señala esta relación positiva.

Ahora bien, en este ámbito hay que tener en cuenta el efecto del déficit público derivado de alguna o de ambas actuaciones. Entre otras cuestiones, ello puede suponer un aumento futuro de los impuestos, con la incidencia negativa que ello supone, un efecto "crowding-out" debido al incremento en el tipo de interés derivado de la emisión de la deuda necesaria para financiar ese déficit, que supondría una menor actividad privada, y un efecto riqueza negativo en lo que se refiere al consumo, ya que los agentes económicos se podrían considerar menos ricos.

Todo lo anterior supondría perjudicar el crecimiento económico, con el efecto a su vez negativo que tendría sobre el emprendedurismo, como hemos indicado anteriormente. El análisis empírico parece corroborar el efecto "feed-back" entre renta y TEA (Galindo, Méndez y Alfaro, 2010), por lo que los efectos que acabamos de indicar afectarían en el futuro negativamente a las iniciativas emprendedoras.

Por lo que se refiere a la política monetaria, ésta también afectaría a los emprendedores, ya que uno de los elementos esenciales que inciden sobre su actividad es la disponibilidad de recursos financieros. Por tanto, una política monetaria expansiva sería beneficiosa, al poner a su disposición más recursos para llevar a cabo su inversión, siendo esto válido tanto para el caso del TEA total (Alfranca, Galindo y Méndez, 2011) como en el referido a las mujeres (M. Bahmani, Galindo y Méndez, 2011).

Ahora bien, este tipo de medida también tiene una serie de efectos indirectos que hay que tener en cuenta. Una política monetaria expansiva generaría tensiones inflacionistas que dañarían la competitividad de los productos y pondría en peligro la senda de crecimiento con las consecuencias que hemos señalado anteriormente. Por otro lado, la reducción de los tipos de interés que ello supondría desestimularía el ahorro, por lo que sería más complicado conseguir los recursos necesarios para la inversión.

Finalmente, hay que considerar una variable adicional, la distribución de la renta. En el modelo schumpeteriano uno de los factores que incentivan la innovación y la actividad empresarial es la existencia de un adecuado clima social. Y, en términos generales, se puede considerar que la distribución de la renta se relaciona con dicho clima, ya que si es adecuada no habrá tensiones sociales que enrarezcan el clima que requieren los emprendedores para llevar a cabo su actividad. El análisis empírico muestra que una mejor distribución de la renta afecta positivamente a la actividad emprendedora, tanto en términos generales (Galindo, Méndez y Alfaro, 
Galindo, Miguel-Ángel y Méndez, María Teresa. La actividad emprendedora y competitividad: factores que inciden sobre los emprendedores.

2010), como en el caso de la mujeres emprendedoras (M. Bahmani, Galindo y Méndez, 2011).

En definitiva, comprobamos cómo a través de las políticas fiscal y monetaria se puede estimular la actividad emprendedora, pero hay que tener en cuenta los efectos indirectos negativos que también generan. Lo importante es que a través de ellas se consiga un importante crecimiento económico que siga estimulando la actividad empresarial y el empleo, ya que gracias a ello se lograrán los recursos necesarios para financiar el déficit ocasionado sin incurrir en los aspectos negativos mencionados.

\section{CONCLUSIONES}

A lo largo de los apartados anteriores hemos expuesto los pilares en los que se fundamenta la competitividad considerada en un sentido amplio. Y uno de los elementos que afectan a la competitividad es la actividad emprendedora, con la que también se consigue aumentar la renta y el bienestar de un país.

El emprendedor es una persona que desarrolla una actividad en la que arriesga una serie de recursos y que ofrece bienes y servicios para satisfacer las necesidades de los agentes económicos. Gracias a su iniciativa se utilizan recursos que estaban ociosos y se alcanza una mayor eficiencia que supone finalmente un mayor crecimiento económico.

Por tanto, debido a la relevancia que tiene este factor, es de gran importancia determinar las medidas que resultan más adecuadas para estimularlo. Con independencia de variables de índole cualitativa, como el capital social o las organizaciones sin ánimo de lucro, las políticas fiscal y monetaria, entre otras, desempeñarían un papel esencial. La primera lo haría a través de las mejoras de infraestructuras y/o las reducciones de impuestos. La segunda, a través de una expansión de la oferta monetaria, lo que supondría mayores recursos financieros para atender las necesidades de inversión que tienen los emprendedores.

Ahora bien, ambos tipos de actuaciones tienen una serie de efectos negativos, como mayor déficit e inflación, que incidirían negativamente sobre los emprendedores y sobre la competitividad. Por ello es necesario que se considere el crecimiento económico que se genera a través de ellas y si los recursos que se consiguen son suficientes para evitar los efectos negativos que acabamos de señalar.

De todas formas, el análisis realizado hasta el momento no es concluyente, siendo necesario ir introduciendo nuevas variables conforme se vaya obteniendo más información estadística y vayan aumentando los años de los que disponemos datos sobre la actividad emprendedora. 
Galindo, Miguel-Ángel y Méndez, María Teresa. La actividad emprendedora y competitividad: factores que inciden sobre los emprendedores.

\section{BIBLIOGRAFÍA}

Acs, Zoltan J., Audretsch, D. B.; Braunerhjelm, P. y Carlson, B. (2004): The missing link the knowledge filter and entrepreneurship in endogenous growth, Working Paper 4783, Center for Economic Policy Research, Londres.

Acs, Zoltan J., Audretsch, D. B., Braunerhjelm, P. y Carlson, B. (2005): Growth and entrepreneurship: an empirical assessment, Working Paper 3205, Discussion Papers on Entrepreneurship, Growth and Public Policy, Max Plank Institute of Economics, Jena, Alemania.

Alfranca, O., Galindo, M. A. y Méndez, M. T. (2011): "Polices to enhance entrepreneurship as an economic growth factor", en Kijanka, E. y Saiz J. M. (Eds.): Global Crisis vs Regional Growth. New European Trends for the XXI Century, School of Economics and Law in Kielce, Polonia (en prensa)

Audretsch, D. B. (2006): The entrepreneurial society, Oxford University Press, Nueva York

Audretsch, D. B. (2009): "The entrepreneurial society", Journal of Technology Transfer. 34 (3), 245-254.

Audretsch, D. B., Bonte, W. y Keilbach, M. (2008): "Entrepreneurship capital and its impact on knowledge diffusion and economic performance", Journal of Business Venturing. 23 (6), 687-698.

Audretsch, D. B. y Keilbach, M.. (2004a): “Does entrepreneurship capital matter?", Entrepreneurship: Theory and Practice. 28 (5), 419-429.

Audretsch, D. B. y Keilbach, M. (2004b). Entrepreneurship Capital and Economic Performance. Regional Studies, 38 (8), 949-959

Audretsch, D. B. y Keilbach, M. (2008): "Resolving the knowledge paradox: Knowledge-spillover entrepreneurship and economic growth", Research Policy. 37 (10), 1697-1705.

Bahmani-Oskooee, M., Galindo, M. A., y Mendez, M. T. (2008): "Social capital and entrepreneurship in a regional context: the case of Spanish region, s”, en M. A. Galindo, Guzmán, J., y Ribeiro, D. (Eds.): Entrepreneurship and Regional Economics, Springer, Berlin, 101-111.

Bahmani-Oskooee, M., Galindo, M. A., y Mendez, M. T. (2011): 'Women's entrepreneurship and economic policies", en M. A. Galindo, y Ribeiro, D. (Eds.): Women's Entrepreneurship and Economics, Springer, Berlin (en prensa).

Bahmani, S., Galindo, M.A. y Mendez, M. T. (2011): "Non Profit Organizations, Entrepreneurship, Social Capital and Economic Growth", Small Business Economics (en prensa).

Baumol, W. J. (1990): "Entrepreneurship: Productive, Unproductive and Destructive", Journal of Political Economy. 80, 893-921.

Birley, S. (1985): "The role of networks in the entrepreneurial process", Journal of Business Venturing. 1, 107-117.

Bull, I., y Willard, G. E. (1993): "Towards a theory of entrepreneurship", Journal of Business Venturing. 8, 183-195.

Galindo, M. A., Méndez, M. T. y Alfaro, J.L. (2010): "Entrepreneurship, income distribution and economic growth", International Entrepreneurship Management Journal. 6, 131-141.

GLOBAL ENTREPRENEURSHIP MONITOR (GEM): Executive Report, varios años.

Harper, D. A. (1998): "Institutional conditions for Entrepreneurship", en Boettke, P. J., Kirzner, I. M. y Rizzo, M. J. (Eds.): Advances in Austrian Economics, vol. 5, JAI Press, Connecticut, 241-275. 
Galindo, Miguel-Ángel y Méndez, María Teresa. La actividad emprendedora y competitividad: factores que inciden sobre los emprendedores.

Herbert, R. F., y Link, A. N. (1989): "In search of meaning of entrepreneurship", Small Business Economics. 1, 39-49.

Holcombe, R. (1998): "Entrepreneurship and Economic Growth", The Quarterly Journal of Austrian Economics. 1, 45-62.

Kirzner, I. M. (1973) : Competition \& Entrepreneurship, University of Chicago Press, Chicago.

Kirzner, I. M. (1999): "Creative and/or alertness: A reconsideration of the Schumpeterian entrepreneur", The Review of Austrian Economics. 11, 5-17.

Knight, F. (1921): Risk, Uncertainty, and Profit, Houghton Mifflin Company, Nueva York.

Lin, N. (2001): "Building a network theory of social capital", en Lin, N., Cook, K., y Burt, R. S. (Eds.): Social Capital. Theory and research, Aldine De Gruyter, Nueva York.

Lumpkin, G. T., y Dess, G. G. (1996): "Clarifying the entrepreneurial orientation construct and linking it to performance", Academy of Management Review. 21, 135-172.

Madrid, M. (2010): "International Competitiveness: Measurement Indicators and Structural Aspects with Specific Reference to the Situation in Spain", en Galindo Martín, M. A. y Nissan, E. (Eds.): International Political Economy, Nova Press, Nueva York, 91-104.

North, D. C. (1994): "Economic performance through time", The American Economic Review. 84, 359-368.

OCDE (1992): Technology and Economy, París.

Olson, M. (1996): "Big bills left on the sidewalk: Why some nations are rich and others poor", Journal of Economic Perspectives. 10, 3-24.

Schumpeter, J. A. (1911): The Theory of Economic Development,Oxford University Press, Nueva York.

Schumpeter, J. A. (1950): Capitalism, Socialism and Democracy, Harper \& Brother Publishers, Nueva York.

Stel, A.; Carree, M.; y Thurik, R. (2005): "The Effect of Entrepreneurial Activity on National Economic Growth", Small Business Economics. 24, (3), 311-321.

Swedberg, R. (2000): "The Social science View of Entrepreneurship", en R. Swedberg (Ed.): Entrepreneurship. The Social Science View, Oxford University Press, Oxford, 7-44.

Weber, M. (1978): Economy and Society, 2 vols, University of California Press, Berkeley.

Weber, M. (1988): The Protestant Ethics and the Spirit of Capitalism, Peter Smith, Gloucester.

Wennekers, A.R.M. y Thurik, A. R. (1999): "Linking Entrepreneurship and economic growth", Small Business Economics.13, 27-55.

WORLD ECONOMIC FORUM (2010): The Global Competitiveness Report, 20102011, Geneva. 